\title{
Sonographically guided lymph node biopsy: Complication rates
}

\author{
Michael Mueller ${ }^{1}$, Genia Wittich ${ }^{1}$, Suemeyra Oeztuerk ${ }^{1}$, Wolfgang Kratzer ${ }^{1}$, Mark Martin Haenle ${ }^{1}$, \\ Richard Andrew Mason ${ }^{2}$
}

${ }^{1}$ Department of Internal Medicine I, University Hospital Ulm, Ulm, Germany

${ }^{2}$ Department of Veterans Affairs, Louis Stokes Cleveland Medical Center, Brecksville, USA

Email: wolfgang.kratzer@uniklinik-ulm.de

Received 23 January 2012; revised 22 February 2012; accepted 21 March 2012

\begin{abstract}
Purpose: The study investigated the rate of complications associated with sonographically guided lymph node biopsies and assessed potential risk factors. Methods: A total of 536 sonographically guided puncture procedures (283 males, $52.8 \%$; 253 females, 47.2\%; average age $57.0 \pm 16.0$ years; range $14-87$ years) were performed in 469 patients for the work-up of unclear lymphadenopathy. Events, complications and potential risk factors, were prospectively documented. Results: The 469 patients underwent a total of 536 puncture procedures (PP) including 663 punctures and 1485 passes. Lymph node localizations were intraabdominal $(55.2 \%, n=296)$, cervical $(22.4 \%, n=$ $120)$, inguinal $(12.9 \%, n=69)$, axillary $(7.8 \%, n=42)$ and other $(1.7 \%, n=9)$. No complications were documented during the entire study period. There was no increased risk of complications documented for the potential risk factors number of punctures, the number of passes, the localization, diameter of the lymph node (s), puncture technique, needle gauge, as well as patients' sex, age and coagulation parameters, and the experience of the examiner. Conclusions: Our findings confirm the safety of percutaneous sonographically guided lymph node biopsies in different regions of the body in patients with adequate coagulation parameters undergoing pre-interventional color Doppler ultrasound examination.
\end{abstract}

Keywords: Lymph Nodes; Complications; Biopsy; Ultrasonography

\section{INTRODUCTION}

Sonographically guided lymph node biopsy is considered a safe and effective diagnostic tool $[1,2]$. It served to exclude or confirm the diagnosis in patients with enlarged lymph nodes and is of decisive clinical value in determining therapy and prognosis [3-5]. Despite real-time imag- ing and pre-interventional assessment of the planned puncture path, complications may very infrequently occur. Complication rates in the literature are in the range of $0 \%-2.9 \%[1-3,6]$. To our knowledge, however, no large studies have addressed the causes of complications in patients undergoing sonographically guided diagnostic lymph node biopsy. The present study prospectively documented complications and required treatments and assessed potential risk factors, including the number of punctures, the number of passes, the localization, diameter of the lymph node (s), puncture technique, needle gauge, as well as patients' sex, age and coagulation parameters, and the experience of the examiner.

\section{MATERIALS AND METHODS}

\subsection{Patient Selection}

A total 4278 diagnostic puncture biopsies (liver, 2280 punctures; lymph nodes, 536 punctures; pancreas, 351 punctures; other locations, 1111 punctures) were performed in 3353 patients in the department of diagnostic ultrasound of our institution between September 1999 and November 2009. The 536 biopsies of lymph nodes were performed in 469 patients aged $14-87$ years (mean 57.0 \pm 16.0 years $) .52 .8 \%(\mathrm{n}=283)$ of the puncture procedures were performed in male patients, $47.2 \%(\mathrm{n}=253)$ in female patients. Of these, $42.7 \%(\mathrm{n}=229)$ of biopsies were performed on an outpatient basis, while $57.3 \%(n=$ 307) were performed on inpatients.

\subsection{Nomenclature}

For the sake of clarity, the present report gives only the outer diameter of puncture needles in millimeters. Assessment considered patients, puncture procedures (PP), punctures and passes.

A PP was defined as an intervention in which a single puncture target was approached through a single puncture channel using one or more puncture needles of the same or different diameter; one or more techniques could 
be used. A puncture was defined as a biopsy using a needle of the same diameter and technique; multiple passes with the same needle were counted as a single puncture. Passes were defined as each entry of the needle into the target region. Thus, throughout the study, individual patients could undergo several PP with numerous punctures, each of these potentially including several passes. Complications were evaluated on the basis of PP as complications occurring during procedures with multiple punctures could not be assigned to any single puncture.

\subsection{Data Collection}

For data collection, a commercially available, PC-based, standardized documentation system (ViewPoint Bildverarbeitung $\mathrm{GmbH}$, Wessling, Germany) whose structured data entry met standards established by the German Society for Ultrasonography in Medicine (DEGUM), was used. The following parameters were systematically recorded in order to calculate their effect on the complication risk: number of punctures, number of passes, sex, age, localization, size, puncture technique, needle diameter, examiner's experience and patient's coagulation status. During the study period, all complications and subsequent therapeutic measures, if any, were documented prospectively in an Excel spread sheet (Microsoft Office Excel 2003).

\subsection{Definition of Complications}

Major complications were defined as post-interventional events that could clearly be attributed to the PP; were clinically relevant; and required therapeutic intervention (e.g. blood transfusion, drainage etc.). Minor complications were events that were not clinically relevant and did not require specific therapeutic intervention. Administration of analgesics was not considered a therapeutic intervention; hence, pain requiring analgesic treatment was regarded as a minor complication.

\subsection{Puncture Requirements}

The following criteria had to be met before each puncture: clear indications with therapeutic consequence; adequate coagulation status (platelet count $\geq 70,000 / \mu 1$, Quick's test value $\geq 70 \%$, partial thromboplastin time (PTT) $<50$ seconds, no application of anticoagulants); and written consent signed by the patient one day prior to the procedure. In rare cases, especially with superficial lymph nodes, punctures were also performed in patients not meeting the required coagulation criteria. This was often indicated in the work-up of leukemic disorders in which the choice of appropriate chemotherapy depended on timely diagnosis. Every puncture performed in patients not meeting coagulation criteria was based on a careful evaluation of the relative benefits and risks of the procedure. Examiner's experience was divided into two categories: Those who had performed a minimum of 150 PP were considered "experienced examiners", whereas those who had performed less than 150 PP were considered "inexperienced examiners".

\subsection{Execution}

All punctures were performed under sonographic guidance (Philips HDI 3000/5000 Sono CT; C5-2 curved array transducers and biopsy guidance for deep lymph nodes; sonographically guided free-hand puncture using a L12-5 parallel transducer head for superficial lymph nodes). $75.7 \%$ of punctures $(n=502)$ were performed using fine needle aspiration cytology (FNAC), while $24.3 \%(n=161)$ involved cutting biopsies (CB).

First, the target lesion was sonographically visualized in the epigastric region. Then, penetration length was determined and routine Doppler ultrasound examination was performed in order to minimize the risk of injury to adjacent organs or vessels. After disinfection of the skin directly above the puncture site, local anesthesia with mepihexal $1 \%$ was induced, followed by intravenous application of piritramide (Dipidolor ${ }^{\mathbb{B}} ; 1-2 \mathrm{ml}=10-20$ $\mathrm{mg}$ ) and metoclopramide (Reglan ${ }^{\circledR} ; 2 \mathrm{ml}=10 \mathrm{mg}$ ), and volume and pulse-oxymetric monitoring prior to the actual puncture. For FNAC of deeper lymph nodes, examiners used biopsy guidance in order to insert a catheter (diameter: $1.3 \mathrm{~mm}$ ) into a small skin incision through which the actual puncture needle (Chiba, diameter: 0.7 $\mathrm{mm}$ ) was inserted and advanced into the mass lesion under sonographic guidance. Once the puncture needle was in place, the assistant actuated the Binder valve, thus generating a vacuum, and dislodged cells from their formation by means of poking and fanning movements before aspirating them into the tube. After the puncture needle had been removed the aspirated cytological material was transferred onto a clean glass slide and several smears were made using the glass coverslip. The aspiration procedure could be repeated several times if inadequate cytological material was collected on the first try. Puncture with FNAC (Chiba, diameter: $0.95 \mathrm{~mm}$ ) did not requiring a leader catheter.

Puncture of superficial lymph nodes was performed in free-hand puncture technique using needles with diameters of $0.3-0.9 \mathrm{~mm}$. For cutting biopsies (CB) a puncture needle (Angiotech, Pro-Mag Biopsy needle, diameter: $1.2 \mathrm{~mm}$ and $1.6 \mathrm{~mm}$ ) was advanced into the mass lesion without a catheter using biopsy guidance similar to the procedure used for FNAC. Once the needle was in place, the assistant triggered the fully automated biopsy pistol (Pro-Mag Ultra) upon signal from the examiner. In case of unsatisfactory results this procedure could be repeated as well. After the puncture kit was removed, the tissue sample of ideally $1.5-2 \mathrm{~cm}$ in length was placed 
into $3.5 \%-3.7 \%$ of formaldehyde solution and sent in for histological evaluation. In case both $\mathrm{CB}$ and FNAC were performed, the FNAC was always performed first. After FNAC, the patient was required to maintain four hours' bed rest including a one hour of sandbag compression (three hours after $\mathrm{CB}$ ) of the puncture site and fasting during the same period. Patients' pulse rate and blood pressure were recorded hourly (every 30 minutes after CB). Following CB, patients underwent a final complete blood count, physical examination by the treating physician and sonographic examination. If all findings were within normal limits, patients were discharged.

\subsection{Statistical Evaluation}

Statistical analyses were performed using the SAS 9.2 statistics software package (SAS Institute Inc., Cary, North Carolina, USA). The data of the 536 PP were evaluated descriptively regarding absolute and relative frequencies, mean, standard deviation, minimum and maximum. A multivariate analysis was not performed since no major complications were recorded in our study.

\section{RESULTS}

No complications, either major or minor, were recorded in the present patient collective. No elevated complication rate could be attributed to any of the potential risk factors such as number of punctures, number of passes, patients' sex, age or coagulation status, tumor localization or size, puncture technique, needle diameter, experience of examiner. The 469 patients recruited for the present study underwent a total 536 puncture procedures including 663 punctures and 1485 passes. The localization of all lymph nodes and the associated number of PP is presented in Figure 1. The exact localization of intra-abdominal lymph nodes is given in Figure 2.

Maximum lymph node diameter was documented in $92.4 \%(\mathrm{n}=495)$ PP. Mean maximum diameter was 30.9 $\pm 24.8 \mathrm{~mm}$ (range: $5.0-300 \mathrm{~mm}$ ). Needles with a diameter of $0.3-1.6 \mathrm{~mm}$ were used for punctures (Figure 3). In $76.5 \%(n=410)$ of PP, only one puncture was performed; of these, $88.8 \%(\mathrm{n}=364)$ were FNAC and $11.2 \%(n=46)$ were CB. In $23.3 \%(n=125)$ of PP, two punctures were performed. Of these, PP included both $\mathrm{CB}$ and FNAC in $91.2 \%(\mathrm{n}=114)$, while, in $8.8 \%(\mathrm{n}=$ 11), patients underwent two FNAC using puncture needles of different diameters. Only in one instance $(0.2 \%, \mathrm{n}$ $=1$ ) did PP consist of three punctures, two of which were FNAC and one, CB. The average number of passes was $1.2 \pm 0.5$ (range: 1 - 3) for $\mathrm{CB}$ and $2.6 \pm 0.9$ (range: 1 - 6) for FNAC (Figure 4).

During the period of the study, a total of 33 different examiners performed a mean $125 \pm 254.6 \mathrm{PP}$ (range 4 $1480)$ on various organs. Overall, $9.1 \%(n=3)$ of the

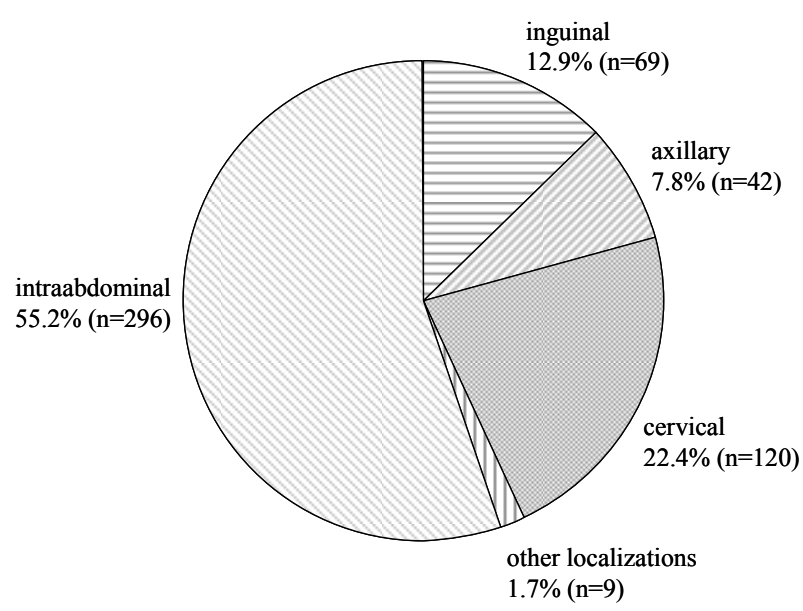

Figure 1. Lymph node localizations.

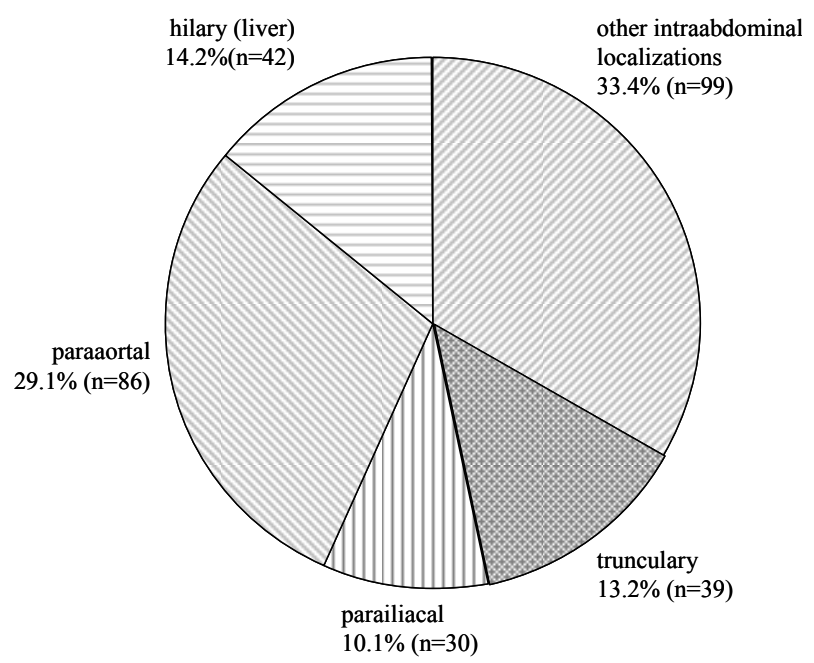

Figure 2. Intraabdominal localizations.

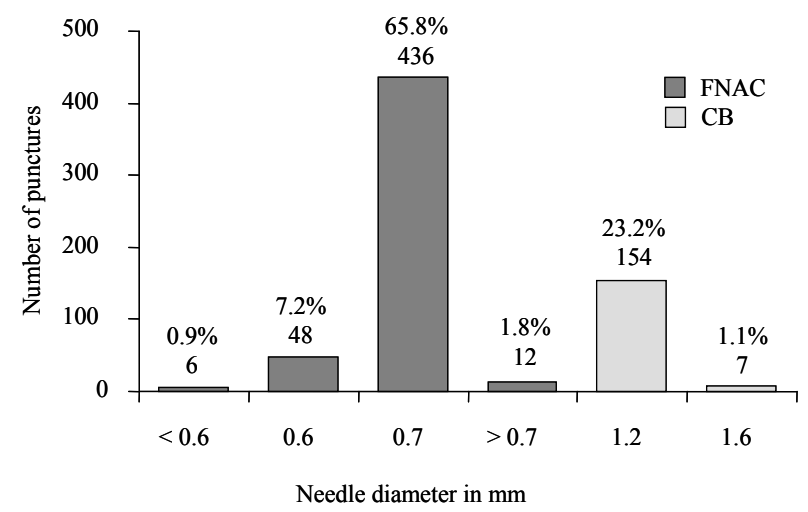

Figure 3. Biopsy type and needle gauge.

examiners reached an "experienced" level during this time, while $90.9 \%(\mathrm{n}=30)$ performed less than $150 \mathrm{PP}$ and were therefore considered "inexperienced". 


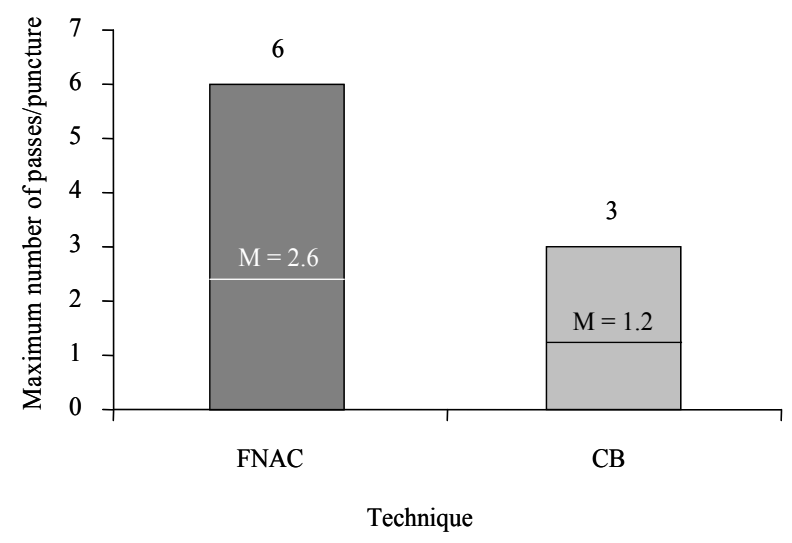

Figure 4. Maximum and mean needle passes per puncture.

Coagulation parameters (platelets, Quick's value, PTT) were documented in $38.6 \%(n=207)$ of all PP. Of these, $90.3 \%(\mathrm{n}=187)$ were performed in patients meeting minimum criteria established for coagulation status, while, in $9.7 \%(n=20)$ criteria were not fully met. Platelet count was documented in $41.6 \%(n=223)$ of the PP and was below the limit of $\geq 70,000 / \mu 1$ in $4.5 \%(n=10)$; the lowest platelet count was $11,000 / \mu 1$. Quick's value was documented in $42.5 \%(n=228)$ of the PP and was below the minimum limit of $\geq 70 \%$ in $6.1 \%(n=14)$ of all cases; the lowest Quick's value registered for a PP was at $32 \%$. PTT was documented in $39.6 \%(n=212)$ of the PP and was above the limit of $<50$ seconds in $1.4 \%$ $(n=3)$ of cases. The highest PTT registered for a PP was 105 seconds. In only one case was PP performed in the presence of abnormal findings for all three coagulation parameters.

\section{DISCUSSION}

Lymph node biopsies are generally considered to be very safe diagnostic procedures [1]. No complications were recorded in the present patient collective. In the literature, reported rates are low, ranging from 0 to $2.9 \%$ [1-10] (Table 1). Major complications were reported in two publications, both secondary to vascular injuries $[3,6]$. The available studies, however, concentrated on the outcome of the punctures; complications were addressed more as a secondary issue that, in some cases, was not specifically discussed $[11,12]$. To our knowledge, no current studies have specifically investigated the complication rate of lymph node punctures. Other studies that have ad-

Table 1. A review of the literature on sonographically guided lymph node biopsies.

\begin{tabular}{|c|c|c|c|c|c|c|c|c|}
\hline Authors & Date & $\begin{array}{l}\text { Complications per } \\
\text { total punctures }\end{array}$ & $\begin{array}{l}\text { Complication } \\
\text { rate in } \%\end{array}$ & $\begin{array}{c}\text { Type of } \\
\text { complication }\end{array}$ & Imaging & $\begin{array}{l}\text { Color } \\
\text { doppler }\end{array}$ & $\begin{array}{l}\text { Coagulation } \\
\text { parameters }\end{array}$ & $\begin{array}{l}\text { Maximum needle } \\
\text { gauge }\end{array}$ \\
\hline $\begin{array}{l}\text { Takashima } \\
\text { et al. [3] }\end{array}$ & 1997 & 1 major/91 & 1.1 & $\begin{array}{l}\text { Puncture of } \\
\text { carotid artery }\end{array}$ & SFHP & $\mathrm{n} / \mathrm{a}$ & $\mathrm{n} / \mathrm{a}$ & $0.7 \mathrm{~mm}$ \\
\hline $\begin{array}{l}\text { Fisher } \\
\text { et al. [6] }\end{array}$ & 1997 & 1 major/35 & 2.9 & $\begin{array}{c}\text { Puncture of } \\
\text { inferior epigastric } \\
\text { artery }\end{array}$ & SGP & Yes & $\mathrm{n} / \mathrm{a}$ & $1.2 \mathrm{~mm}$ \\
\hline $\begin{array}{l}\text { Screaton } \\
\text { et al. [1] }\end{array}$ & 2002 & 3 minor/260 & 1.2 & 3 hematomas & SFHP & Yes & No & $1.6 \mathrm{~mm}$ \\
\hline $\begin{array}{l}\text { Wittich } \\
\text { et al. }\end{array}$ & 2010 & $0 / 663$ & 0 & - & $\begin{array}{l}\text { SGP/ } \\
\text { SFHP }\end{array}$ & Yes & Yes & $1.6 \mathrm{~mm}$ \\
\hline 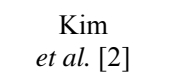 & 2007 & $0 / 155$ & 0 & - & SFHP & Yes & No & $1.6 \mathrm{~mm}$ \\
\hline $\begin{array}{l}\text { Cheung } \\
\text { et al. [4] }\end{array}$ & 2000 & $0 / 60$ & 0 & - & SFHP & $\mathrm{n} / \mathrm{a}$ & $\mathrm{n} / \mathrm{a}$ & $1.2 \mathrm{~mm}$ \\
\hline $\begin{array}{c}\text { Abe } \\
\text { et al. [7] }\end{array}$ & 2007 & $0 />100$ & 0 & - & SFHP & Yes & $\mathrm{n} / \mathrm{a}$ & $2.1 \mathrm{~mm}$ \\
\hline al-Mofleh [8] & 1992 & $0 / 240$ & 0 & - & SPU & $\mathrm{n} / \mathrm{a}$ & $\mathrm{n} / \mathrm{a}$ & $0.9 \mathrm{~mm}$ \\
\hline $\begin{array}{l}\text { Veerapand } \\
\text { et al. [10] }\end{array}$ & 2004 & $0 / 72$ & 0 & - & SFHP & $\mathrm{n} / \mathrm{a}$ & $\mathrm{n} / \mathrm{a}$ & $0.9 \mathrm{~mm}$ \\
\hline $\begin{array}{l}\text { Nagano } \\
\text { et al. [5] }\end{array}$ & 1991 & $0 / 16$ & 0 & - & SGP & $\mathrm{n} / \mathrm{a}$ & $\mathrm{n} / \mathrm{a}$ & $0.8 \mathrm{~mm}$ \\
\hline $\begin{array}{l}\text { Memel } \\
\text { et al. [9] }\end{array}$ & 1996 & $0 / 26$ & 0 & - & SGP & Yes & Yes & $1.2 \mathrm{~mm}$ \\
\hline
\end{tabular}

SFHP: Sonographic free-hand puncture, SGP: Sonographically guided puncture, SPU: Sonographic puncture of unknown modality, n/a: Not available. 
dressed complications have reported smaller series of 16 to 260 lymph node punctures without clear description of how the data were gathered $[3,6]$.

Because there were no complications reported in the present study, it was not possible to show an increased risk of complications for the potential risk factors of number of punctures, the number of passes, the localization, diameter of the lymph node (s), puncture technique, needle gauge, as well as patients' sex, age and coagulation parameters, and the experience of the examiner. To our knowledge, no other study has included an analysis of this type, which, because of the often small number of punctures and complications, is not always feasible [1, $3,6]$.

We attribute our low complication rate to the fact that all punctures were performed under sonographic realtime guidance and that we routinely obtained pre-interventional color Doppler ultrasound examination and coagulation screening. To date, color Doppler ultrasound examination and routine coagulation screening have yet to be established in the standard pre-interventional work-up $[3,5,6,8,10]$ (Table 1). Based on the current literature, and supported by the findings of the present study, we recommend obtaining a color Doppler ultrasound examination prior to every planned lymph node biopsy: this includes cases of both superficial lymph nodes and nodes that would appear to be easily distinguished from blood vessels. Further, we recommend routine screening of patients' coagulation status. Quick's test of $70 \%$ and platelet count of $70,000 / \mu 1$ appear to be justified and contribute to maintaining low complication rates. In cases of superficial lymph nodes, a decision to perform a puncture in patients not meeting the above coagulation criteria might be made on the basis of a careful benefit-torisk consideration.

In the United States, CT is considered the imaging method of choice for guided puncture of intra-abdominal structures [9]. Advantages of CT over ultrasound include the superior anatomical visualization, the capacity for detailed delineation of the target lesion from surrounding structures, uniform viewing conditions not affected by the presence of intestinal gas and more exact visualizetion of the puncture needle [6]. With respect to the success rate for biopsies, however, ultrasound and CT are comparable [9]. In fact, Takashima et al. [3] presents data that suggests that ultrasound is superior to CT and MRI. Complication rates for CT-guided punctures are reported in the range of $0.05 \%-2.5 \%$ [13], which is comparable to complication rates reported for sonographically guided punctures $(0 \%-2.9 \%)$ [1-10]. A comparison study reported by Sheafor et al. [14] also found no significant difference in the rate of complications between CT- and sonographically guided punctures. Be- cause of the capacity for real-time visualization of both needle placement and the entire puncture procedure, as well as more rapid and mobile application, absence of radiation exposure and cost efficiency, ultrasound would appear to be the imaging method of choice for guided lymph node puncture; with comparable results and a similar complication rate it is a sensible alternative to CT-guided punctures. Our findings support Memel et al. [9], who concluded that ultrasound is the imaging method of choice for guided puncture of intra-abdominal lymph nodes.

The findings of the present study are in accord with reports in the current literature which show conclusively that percutaneous sonographically guided lymph node puncture biopsy is a very safe procedure. Routine preinterventional color Doppler ultrasound and coagulation screening minimizes the risk of serious bleeding complications. As an imaging method for puncture guidance, $\mathrm{CT}$ and ultrasound exhibit comparable success and complication rates. Given the absence of radiation exposure, real-time visualization and cost efficiency, we conclude that ultrasound should be considered the imaging method of choice for guided puncture of lymph nodes. Further studies are needed to develop evidence-based guidelines that with further minimize the risk of complications and enhance the safety of patients undergoing sonographically guided puncture.

\section{REFERENCES}

[1] Screaton, N.J., Berman, L.H. and Grant, J.W. (2002) Head and neck lymphadenopathy: Evaluation with USguided cutting-needle biopsy. Radiology, 224, 75-81. doi:10.1148/radiol.2241010602

[2] Kim, B.M., Kim, E.K., Kim M.J., et al. (2007) Sonographically guided core needle biopsy of cervical lymphadenopathy in patients without known malignancy. Journal of Ultrasound in Medicine, 26, 585-591.

[3] Takashima, S., Sone, S., Nomura, N., et al. (1997) Nonpalpable lymph nodes of the neck: Assessment with US and US-guided fine-needle aspiration biopsy. Journal of Clinical Ultrasound, 25, 283-292.

doi:10.1002/(SICI)1097-0096(199707)25:6<283::AID-JC $\underline{\mathrm{U} 1>3.0 . \mathrm{CO} ; 2-8}$

[4] Cheung, Y.C., Wan, Y.L., Lui, K.W. and Lee, K.F. (2000) Sonographically guided core-needle biopsy in the diagnosis of superficial lymphadenopathy. Journal of Clinical Ultrasound, 28, 283-289. doi:10.1002/1097-0096(200007/08)28:6<283::AID-JCU3 $>3.0 . \mathrm{CO} ; 2-\mathrm{T}$

[5] Nagano, T., Nakai, Y., Taniguchi, F., et al. (1991) Diagnosis of paraaortic and pelvic lymph node metastasis of gynecologic malignant tumors by ultrasound-guided percutaneous fine-needle aspiration biopsy. Cancer, 68, 2571-2574.

doi:10.1002/1097-0142(19911215)68:12<2571::AID-CN CR2820681207>3.0.CO;2-9

[6] Fisher, A.J., Paulson, E.K., Sheafor, D.H., Simmons, C.M. 
and Nelson, R.C. (1997) Small lymph nodes of the abdomen, pelvis, and retroperitoneum: Usefulness of sonographically guided biopsy. Radiology, 205, 185-190.

[7] Abe, H., Schmidt, R.A., Sennett, C.A., Shimauchi, A. and Newstead, G.M. (2007) US-guided core needle biopsy of axillary lymph nodes in patients with breast cancer: Why and how to do it. RadioGraphics, 27, S91-S99. doi: $10.1148 / \mathrm{rg} .27 \mathrm{si} 075502$

[8] Al-Mofleh, I.A. (1992) Ultrasound-guided fine needle aspiration of retroperitoneal, abdominal and pelvic lymph nodes. Diagnostic reliability. Acta Cytology, 36, 413-415.

[9] Memel, D.S., Dodd, G.D. III and Esola, C.C. (1996) Efficacy of sonography as a guidance technique for biopsy of abdominal, pelvic, and retroperitoneal lymph nodes. American Journal of Roentgenology, 167, 957- 962.

[10] Veerapand, P., Chotimanvijit, R., Laohasrisakul, N. and Muennooch, W. (2004) Percutaneous ultrasound-guided fine needle aspiration of abdominal lymphadenopathy in AIDS patients. Journal of the Medical Association of Thailand, 87, 400-404.
[11] Kline, T.S., Kannan, V. and Kline, I.K. (1984) Lymphadenopathy and aspiration biopsy cytology. Review of 376 superficial nodes. Cancer, 54, 1076-1081. doi:10.1002/1097-0142(19840915)54:6<1076::AID-CNC R2820540624>3.0.CO;2-W

[12] Lioe, T.F., Elliott, H., Allen, D.C. and Spence, R.A. (1999) The role of fine needle aspiration cytology (FNAC) in the investigation of superficial lymphadenopathy; uses and limitations of the technique. Cytopathology, 10, 291297. doi:10.1046/j.1365-2303.1999.00183.x

[13] Oyen, R.H., Van Poppel, H.P., Ameye, F.E., et al. (1994) Lymph node staging of localized prostatic carcinoma with CT and CT-guided fine-needle aspiration biopsy: Prospective study of 285 patients. Radiology, 190, 315322.

[14] Sheafor, D.H., Paulson, E.K., Simmons, C.M., De Long, D.M. and Nelson, R.C. (1998) Abdominal percutaneous interventional procedures: Comparison of CT and US guidance. Radiology, 207, 705-710. 